\title{
PERSPECTIVA Y ANÁLISIS DE GÉNERO EN LAS INVESTIGACIONES SOBRE MOVIMIENTOS SOCIALES Y FEMINISMOS EN EL CONTEXTO ESPAÑOL: UNA REVISIÓN SISTEMÁTICA
}

\author{
GENDER PERSPECTIVE AND ANALYSIS IN SOCIAL \\ MOVEMENT AND FEMINISMS RESEARCH IN THE SPANISH \\ CONTEXT: A SYSTEMATIC REVIEW
}

Lorena MORÁN-NECHES y Julio RODRÍGUEZ-SUÁREZ

Authors / Autores:

Lorena Morán-Neches

Universidad de Oviedo

Oviedo, Spain

moranecheslorena@gmail.com

https://orcid.org/0000-0003-1089-3378

Julio Rodríguez-Suárez

Universidad de Oviedo

Oviedo, Spain

rodriguezsjulio@uniovi.es

https://orcid.org/0000-0003-4373-8905

Submitted / Recibido: 07/05/2021

Accepted / Aceptado: 01/09/2021

To cite this article / Para citar este artículo: Morán-Neches, L. y Rodríguez-Suárez, J. (2022). Perspectiva y análisis de género en las investigaciones sobre movimientos sociales y feminismos en el contexto español: una revisión sistemática. Feminismo/s, 39, 211-240. https://doi.org/10.14198/ fem.2022.39.08

\section{Licence / Licencia:}

This work is licensed under a Creative Commons Attribution 4.0 International.

\section{(c) (1)}

(C) Lorena Morán-Neches y Julio Rodríguez-Suárez

\section{Resumen}

A pesar de que en los últimos años la movilización social se ha venido constituyendo como un importante campo de estudio para las Ciencias Sociales en general, y para la Sociología en particular, y pese a la relevancia que el activismo feminista ha ido adquiriendo hasta conformarse en la actualidad como uno de los principales movimientos a nivel internacional, no existen en el contexto español revisiones bibliográficas de investigaciones sobre esta temática desarrolladas desde una perspectiva de género. Para paliar en la medida de lo posible esta situación, se ha llevado a cabo una revisión sistemática sobre la perspectiva de género y el análisis de los feminismos en el estudio de los movimientos sociales en nuestro país. De las 101 investigaciones empíricas que cumplían los criterios de inclusión (estudios primarios publicados en las principales revistas españolas de Sociología entre 2010 y 2019), fueron seleccionadas las 19 que abordan de manera central la cuestión de género y los feminismos. Los resultados del análisis de estas investigaciones evidencian la pluralidad 
y diversidad temática, el uso casi exclusivo de metodología cualitativa, la ausencia de estudios con finalidad interventiva o de aplicación práctica, así como el creciente interés por conocer las nuevas dinámicas, canales y formas particulares de acción que presenta este tipo de activismo, como los feminismos en red o ciberfeminismos, cuyo auge plantea la dicotomía entre la movilización online y la acción social feminista en la calle. Por último, se destaca la necesidad tanto de incluir el género de forma transversal en el análisis de la movilización, como de atender en concreto a la dimensión psicosocial de la participación femenina.

Palabras clave: revisión sistemática; investigaciones empíricas; movimientos sociales; género; feminismos.

\begin{abstract}
Despite the fact that in recent years social mobilization has become an important field of study for the Social Sciences in general, and for Sociology in particular, and despite the relevance that the feminist activism has been acquiring until it has become one of the main movements at an international level, there are no bibliographical reviews of research on this subject carried out from a gender perspective in the Spanish context. In order to alleviate this situation as far as possible, a systematic review on the gender perspective has been carried out and social movements in our country has been analysed. Of the 101 empirical studies that met the criteria for inclusion (primary studies published in major Spanish journals of sociology between 2010 and 2019) 19 were selected that dealt centrally with the issue of gender and feminism. The results of the analysis of these studies show the plurality and thematic diversity, the almost exclusive use of qualitative methodology, the absence of studies with an interventional or practical application purpose, as well as the growing interest in understanding the new dynamics, channels and particular forms of action presented by this type of activism, such as networked feminisms or cyberfeminisms, whose rise raises the dichotomy between online mobilisation and feminist social action in the street. Finally, the need to include a transversal approach to gender in the analysis of mobilisation is highlighted, as well as the need to pay specific attention to the psychosocial dimension of women's participation.
\end{abstract}

Keywords: systematic review; empirical studies; social movements; gender; feminisms. 


\section{INTRODUCCIÓN Y ESTADO DE LA CUESTIÓN}

\subsection{Movimientos sociales y su desarrollo en el contexto español}

Los movimientos sociales surgen cuando la percepción de injusticia ante una situación concreta genera un conjunto de valores compartidos, basados en la solidaridad, que orientan a la movilización social con intención de cambiarla (Javaloy, 2003). Su aparición y desarrollo, desde posiciones progresistas, pero también conservadoras, fortalece el espacio público y revitaliza la sociedad civil (Tejerina, 1998), pudiendo contribuir a largo plazo a la democratización de las sociedades (Tilly, 2005).

A pesar de la complejidad de su conceptualización, dado su carácter heterogéneo y los difusos límites que comparte con otras formas de acción política (Pérez Ledesma, 1994), numerosos/as autores/as han venido identificando o refiriéndose a la movilización social como herramienta de transformación de la realidad, es decir, de cambio social o conquista de derechos, basada en la acción colectiva a partir de un conflicto dado. Sin embargo, no existe una lógica universal de la acción colectiva ni, en suma, de los movimientos sociales, sino que cada contexto, cada conflicto y cada situación de partida dan lugar a muy diferentes formas de acción y movilización social. Todas ellas pueden llegar a conformar un factor desencadenante de cambios sociales multicausales a largo plazo (Aguilar, 2001; Díez, 2019), tanto en su impacto social en las políticas institucionales, como en la transformación de subjetividades y comportamientos a nivel colectivo e individual (Pleyers y Álvarez-Benavides, 2019). Al mismo tiempo, no se puede olvidar que la movilización social tiene consecuencias de carácter endógeno y cultural (Laraña, 1999) e influye en los procesos psicosociales de las personas que forman parte de ella (Íñiguez, 2003).

Es desde esta óptica multifactorial desde donde debe ser analizado el estudio de los movimientos sociales, constituido ya como un campo propio de investigación, teniendo en cuenta su indudable pluralidad, sus aspectos estructurales y dinámicos, su influencia a nivel macro y microsocial, así como las particularidades tanto geográficas como temporales de los contextos donde tienen lugar. En el caso español, en concreto, las investigaciones surgidas a finales de la década de los setenta, de carácter fundamentalmente 
teórico y descriptivo (Pont Vidal, 1998), dejan paso, a principios del siglo XXI, al estudio más sistematizado y empírico de los movimientos sociales, en especial a partir de la globalización de las movilizaciones. Las manifestaciones del 15 de mayo de 2011, del movimiento 15M y de los indignados, proporcionan un espacio más amplio a reivindicaciones relacionadas con la vivienda, la ecología o el movimiento LGTBI, al tiempo que otorgan mayor visibilidad a las luchas feministas y sus demandas, que eclosionarán en la huelga feminista global del 8M, instaurada en nuestro país en 2018. Estos nuevos movimientos sociales, más globales, digitalizados y horizontales, presentan particularidades tanto en su confección, organización y desarrollo como en la combinación de lo virtual con lo material, requiriendo de nuevas claves interpretativas y metodologías de análisis actualizadas. Esto parece darle un impulso a su estudio, si bien no existe en los últimos años ninguna revisión que aborde de modo genérico las investigaciones sobre movimientos sociales en el contexto español, ni menos aún que centre su análisis en el género y/o los feminismos.

\subsection{Género, feminismos y movimientos sociales}

Resulta fundamental comenzar recordando el papel particular de las mujeres dentro de los movimientos sociales, invisibilizado y limitado en buena medida a lo largo de la historia al haber sido relegadas a la esfera privada de la familia y la domesticidad, generando un doble reduccionismo donde se despolitiza lo doméstico y se restringe la política a lo público (Di Liscia, 2007). En los momentos en los que se ha producido, progresivamente, una cierta apertura (para el caso particular de España, véase Grau Biosca, 1993; Folguera, 1998; o Ramos, 2000), ésta no ha dejado de ser igualmente parcial, convirtiendo determinados espacios sociales en «femeninos» y manteniendo otros, como el ámbito político, bajo la categoría de «masculino», vetando formal o informalmente su acceso a las mujeres.

A pesar de la enorme expansión que el movimiento feminista ha alcanzado en los últimos tiempos, introduciéndose en las instituciones (Goldsmith, 2002) y diluyendo sus límites en el conjunto del ideario social común, parece indiscutible que la desigualdad sigue presente y que dimensiones sociales como la movilización continúan marcadas por sesgos de género, pues la 
participación, en este caso en movimientos sociales, no deja de ser una construcción social normativizada. Por una parte, las mujeres no cuentan con las mismas posibilidades ni oportunidades de «acceso» que los hombres, ya que el tiempo del que disponen para ello y su gestión son realmente diferentes (Sagastizabal y Luxán, 2016), dada su mayor carga de responsabilidades derivadas del ámbito doméstico y de cuidados. Por otro lado, la participación social femenina se encuentra sesgada por unas relaciones de poder desequilibradas y por la existencia de diversas creencias estereotipadas alrededor del género y la tradicional imagen de feminidad y masculinidad, haciendo que sean percibidas, por ejemplo, las formas más «radicales» o «violentas» de participación como propias del género masculino, o algunos ámbitos de participación como el comunitario como más propios del género femenino. De hecho, estas relaciones de poder y dominación no solamente forman parte de la estructura de los movimientos sociales, sino que también influyen en las propias relaciones de quienes participan en ellos, pudiendo convertirse, así mismo, en agentes de producción de las lógicas patriarcales (Dunezat, 2017).

Todo ello hace que sea fundamental dedicar un espacio propio a la cuestión del género dentro del estudio de los movimientos sociales, así como tomar como objeto de análisis la movilización femenina y sus particularidades, tanto en sus significados como en sus materializaciones prácticas. Así, incorporar una perspectiva de género en la investigación implica tener esta variable en cuenta durante todo el proceso (Ministerio de Ciencia e Innovación, 2011), sean su objeto de estudio la movilización femenina y feminista o los movimientos sociales de forma general, observando el papel que mujeres y hombres cumplen en ellos.

En la actualidad puede considerarse a los feminismos como una corriente teórica (Nicolás, 2009) e incluso como una perspectiva metodológica para la investigación (Keller, 1991), pero continúa siendo un movimiento social eminentemente práctico con un claro objetivo transformador por el que ha terminado por consolidarse como el movimiento social que más participación femenina aúna. Se trata, ante todo, de un movimiento político colectivo que surge de la relación y del diálogo con otras personas, del sentimiento de injusticia ante una construcción social desigual que invisibiliza, discrimina y 
violenta a las mujeres por el simple hecho de serlo, así como de la aspiración colectiva a alcanzar una sociedad justa e igualitaria. En palabras de Ahmed (2018), «el feminismo es necesario por todo aquello que no ha acabado: el sexismo, la explotación sexual y la opresión sexual» (p. 18). Partiendo de la diversidad de perspectivas que pueden englobarse bajo este término, así como de los diferentes aspectos en los que sus luchas pueden incidir, nos referiremos a los feminismos de una manera plural para evidenciar la heterogeneidad interna que le caracteriza como movimiento social global que reúne muy diversas identidades, posiciones y reivindicaciones (Galdón, 2019).

De este modo, lo particular y lo general se interrelacionan dentro de una dinámica feminista plural en la que se van construyendo, sobre las pequeñas conexiones, los espacios de encuentro y reunión que permiten el reconocimiento de una realidad desigual común y la identificación con una lucha para cambiarla. Precisamente es por su aspiración a transformar una estructura social tan fuertemente arraigada por lo que el feminismo precisa nacer de lo cotidiano e inundar todos los ámbitos y dimensiones de la sociedad para conformarse transversal.

Dicha transversalidad tiene importantes consecuencias para el acercamiento al estudio de los feminismos y sus formas de hacer, pues, debido a la progresiva instalación de esta corriente en la academia, cada vez es más habitual que se ubique la producción de conocimiento en estos espacios, invisibilizando las iniciativas y el trabajo que tienen lugar en el seno de los colectivos sociales (Pantera Rosa, 2004). Esto genera imágenes estancas de los movimientos, construidas por quienes se dedican a la investigación social, que hace que se les conciba únicamente en su dimensión para la acción y se asigne a actores externos la tarea de teorizar y producir conocimiento a partir de ello. Romper esta tradicional dicotomía entre academia y activismo implica considerar necesaria la interrelación entre teoría feminista y movilización social colectiva (Pajares, 2020).

Por otro lado, a la acción social feminista en las calles hay que sumarle su creciente presencia en los espacios virtuales, nuevos lugares para la participación y socialización de una forma mucho más inmediata, accesible y masiva. Estos nuevos entornos proponen retos a las formas tradicionales de acción de los movimientos, debiendo adaptarse a las nuevas dinámicas que 
el ciberactivismo incorpora. Partiendo de que la expansión del uso de las nuevas tecnologías ha favorecido la aparición de nuevas formas de expresión en los movimientos sociales, multiplicando el número y la diversidad de sus espacios de acción y protesta a través de la red, se puede hablar del ciberfeminismo como una forma de movilización que, si bien comparte muchos de los elementos del movimiento social del que parte, cuenta ya con sus propios espacios y lenguajes para desarrollar el activismo feminista en estos contextos virtuales (Astudillo-Mendoza et al., 2020).

\section{MÉTODO}

Partiendo de la premisa de que las aplicaciones de los trabajos empíricos resultan no sólo pertinentes sino también necesarias tanto para describir, explicar y comprender las demandas, acciones y procesos que desencadenan los movimientos sociales, como para profundizar en los tópicos asociados al comportamiento colectivo (Adell, 2000), nuestro estudio se plantea la siguiente pregunta de investigación:

- ¿En qué medida la investigación sociológica está incorporando la perspectiva de género y de qué manera está analizando los feminismos en el estudio de los movimientos sociales en el contexto español?

A partir de este objetivo principal, se establecen los siguientes objetivos específicos:

- Conocer el espacio que ocupan las investigaciones realizadas desde la perspectiva de género en el total de estudios sobre los movimientos sociales realizados en la última década en el contexto español.

- Analizar las aproximaciones metodológicas utilizadas en dichos estudios empíricos.

- Conocer las temáticas abordadas y las principales conclusiones de estas investigaciones, ahondando en el análisis de los feminismos.

- Profundizar en las particularidades del ciberfeminismo y su abordaje por parte de la investigación sociológica. 
Para integrar de forma objetiva y ordenada los estudios sobre movimientos sociales desarrollados en España durante los últimos años, en concreto aquellos llevados a cabo con perspectiva y/o análisis de género, así como los que tienen entre su objeto de estudio la movilización feminista, se decidió efectuar una revisión sistemática de la literatura científica, una metodología rigurosa para efectuar el proceso de revisión de la investigación en un campo específico de conocimiento (Sánchez-Meca, 2010) que permite sintetizar los resultados de investigaciones primarias utilizando estrategias que limitan el sesgo y el error aleatorio (Cook et al., 1995). La finalidad de esta revisión consiste en analizar la utilización de la perspectiva y el análisis de género por parte de las investigaciones analizadas, entendiendo por ello la presencia de un interés particular en visibilizar y conocer aquellas dinámicas, roles o tendencias de participación diferenciales en mujeres y hombres y derivadas de su género, y profundizar en las principales aproximaciones metodológicas utilizadas, así como en las temáticas y hallazgos más relevantes de los estudios de género y/o feministas.

\subsection{Procedimiento}

La revisión sistemática se realizó adaptando a nuestro caso concreto los estándares PRISMA relativos al diseño metodológico en cuanto a protocolo, proceso de búsqueda, selección y síntesis de resultados (Moher et al., 2009; Urrútia y Bonfill, 2010). A fin de responder a la pregunta de investigación, en un primer momento se utilizó la estrategia PICoS (Pertegal-Vega et al., 2019) para definir los criterios de elegibilidad (población, fenómeno de interés, contexto y diseño del estudio), escogiéndose los siguientes criterios de inclusión para la selección de estudios relevantes:

- Investigaciones empíricas sobre movimientos sociales (temática general o concreta).

- Estudios primarios: utilización de metodología propia.

- Análisis del ámbito español.

- Publicación en los últimos diez años (2010-2019). 
- Publicación en las principales revistas científicas españolas de Sociología.

A pesar de aumentar la complejidad del proceso, se decidió no poner en marcha, como suele ser habitual, una búsqueda general en bases de datos, sino acudir directamente a las propias revistas científicas para localizar aquellas de mayor impacto dentro del ámbito de las Ciencias Sociales (y en particular de la Sociología), reconociendo que, al menos en cierta medida, la calidad de la investigación está relacionada con la calidad de las publicaciones en las que aparece (Requena, 2014). Al hacerlo, asumimos desde un primer momento las limitaciones que, por otra parte, también esta decisión conlleva, tales como las críticas que en ocasiones suscita la forma de selección de los estándares de calidad de las revistas (Delgado-López-Cózar, 2020), las variaciones en la calidad de las revistas seleccionadas a lo largo de los diez años analizados, o la pérdida de información relevante al no incorporar publicaciones en otros formatos como libros, capítulo de libro, tesis, etc.

Para decidir qué revistas analizar, en función de su calidad bajo estándares de fuentes oficiales, se tomó como referencia, en primer lugar, su inclusión en la edición de 2019 de Journal Citation Reports (JCR), encontrándose 5 revistas ${ }^{1}$. Posteriormente, acudimos al Ranking de Visibilidad e Impacto de Revistas Científicas Españolas de Humanidades y Ciencias Sociales con sello de calidad FECYT (Sanz-Casado et al., 2020), que en su edición de 2020 incluye, dentro del área de Ciencias Políticas y Sociología, 34 revistas (4 de ellas seleccionadas previamente en nuestro análisis como JCR)². Además,

1. REIS. Revista Española de Investigaciones Sociológicas (incluida en el área Sociology); RES. Revista Española de Sociología (Sociology); Historia y Política (Political Science); Revista de Estudios Políticos (Political Science); Comunicar. Revista Científica Iberoamericana de Comunicación y Educación (Education \& Educational Research y Communication; incluida además en el ranking FECYT 2019 dentro del área de Ciencias Sociales).

2. América Latina Hoy; Arbor; Areas. Revista Internacional de Ciencias Sociales; Ciudad y Territorio. Estudios Territoriales; Cuadernos de Trabajo Social; Digithum; EMPIRIA. Revista de Metodología de Ciencias Sociales; Encrucijadas. Revista Crítica de Ciencias Sociales; Gestión y Análisis de Políticas Públicas; IDP. Revista de Internet, Derecho y Política; Investigaciones Feministas; Masculinities and Social Change; Methaodos. Revista 
se decidió añadir las 5 revistas con relación temática incluidas en el listado ESCI (Emerging Sources Citation Index) con el criterio de pertenencia al área de Sociología e inclusión en Latindex ${ }^{3}$. De este modo, finalmente fueron 40 las revistas seleccionadas.

El proceso de búsqueda tuvo lugar entre los meses de febrero y mayo de 2020. En una primera fase se creó una base general de todas las referencias sobre investigaciones empíricas de movimientos sociales seleccionadas, mientras que posteriormente, y para el objeto de estudio de este artículo, se realizó una búsqueda específica en dicha base de los estudios relacionados con el género.

El rastreo inicial se llevó a cabo en cada revista a partir de los siguientes términos clave, relacionados con los temas principales del estudio: «movimientos sociales», «movimiento social», «movilización», «movilizaciones», «acción colectiva», «participación social», «activismo»y «protesta».

En la primera fase de la revisión pudimos observar el gran volumen de publicaciones teóricas existente en materia de movimientos sociales en comparación con el número de investigaciones empíricas. Una vez descartados los de carácter teórico, se encontraron un total de 171 artículos de acuerdo con los criterios de selección, que fueron evaluados de manera individual a fin de asignar una ponderación adecuada a las características y resultados obtenidos en cada uno de ellos (Higgins y Green, 2011). De los 171 artículos se desecharon 70: 24 por no ser el análisis de los movimientos sociales su principal objeto de investigación, 4 por tratarse de manifiestos o declaraciones de los propios colectivos, eventos o agrupaciones, 8 por analizar

de ciencias sociales; Migraciones; OBETS. Revista de Ciencias Sociales; Papeles del CEIC. International Journal on Collective Identity Research; Papers. Revista de Sociología; Política y Sociedad; Relaciones Internacionales; Res Publica. Revista de Historia de las Ideas Políticas; Revista CIDOB d'Afers Internacionals; Revista de Estudios Internacionales Mediterráneos; Revista de Paz y Conflictos; Revista Española de Ciencia Política (RECP); Revista Española de Discapacidad (REDIS); Revista Iberoamericana de Ciencia, Tecnología y Sociedad - CTS; Revista Internacional de Sociología; Sociología del Trabajo; Teknokultura: Revista de Cultura Digital y Movimientos Sociales; adComunica. Revista Científica de Estrategias, Tendencias e Innovación en Comunicación.

3. Athenea Digital. Revista de pensamiento e investigación social; Aposta. Revista de Ciencias Sociales; Gazeta de Antropología; Prisma Social; Sociología y Tecnociencia. Revista digital de sociología del sistema tecnocientífico. 
un espectro temporal anterior al establecido en los criterios de análisis, 12 por utilizar únicamente fuentes secundarias, 4 por no aportar información sobre la metodología aplicada, y 18 por debilidad metodológica (muestras muy reducidas, escasa justificación de los criterios de diseño y selección muestral). De esta forma, la selección general estuvo constituida finalmente por 101 artículos.

Una vez finalizada esta primera búsqueda, las 101 referencias bibliográficas fueron exportadas a un archivo Excel donde se codificaron las siguientes variables de cada artículo: autores/as, número de autores y autoras, investigador/a activista o no, año de publicación, revista, temática/s analizada/s, utilización o no de perspectiva de género, el género como objeto de estudio central o tangencial, tipo de estudio, área geográfica estudiada, período temporal analizado, metodología, técnicas de investigación, tamaño de la/s muestra/s, y otra información de interés.

Por último, en la segunda fase de la revisión se procedió a cribar los estudios relacionados con el género o que indicaran la inclusión de dicha dimensión en su análisis, que resultaron ser 28, de los cuales 9 fueron eliminados por no contar con esta variable de manera central y transversal a toda la investigación, configurando una muestra final de 19 documentos (en el siguiente enlace puede consultarse la base de datos).

Los diferentes resultados relacionados con la pregunta de investigación y los objetivos del estudio fueron analizados mediante una red semántica generada con ATLAS.ti v.8., codificándose y categorizándose las unidades de información más relevantes de los artículos seleccionados, a partir de diferentes variables objeto de estudio, tales como «Activismo/s», «Agenda mediática», «Ciberfeminismo», «Conflicto/s», «Discurso/s», «Empoderamiento», «Identidad/es», «Interseccionalidad», «Participación», «Poder/liderazgos», «Público/privado», «Resistencia/s» o «Violencia/s». 
Perspectiva y análisis de género en las investigaciones sobre movimientos sociales y feminismos en el contexto español: una revisión sistemática

Figura 1. Diagrama de flujo

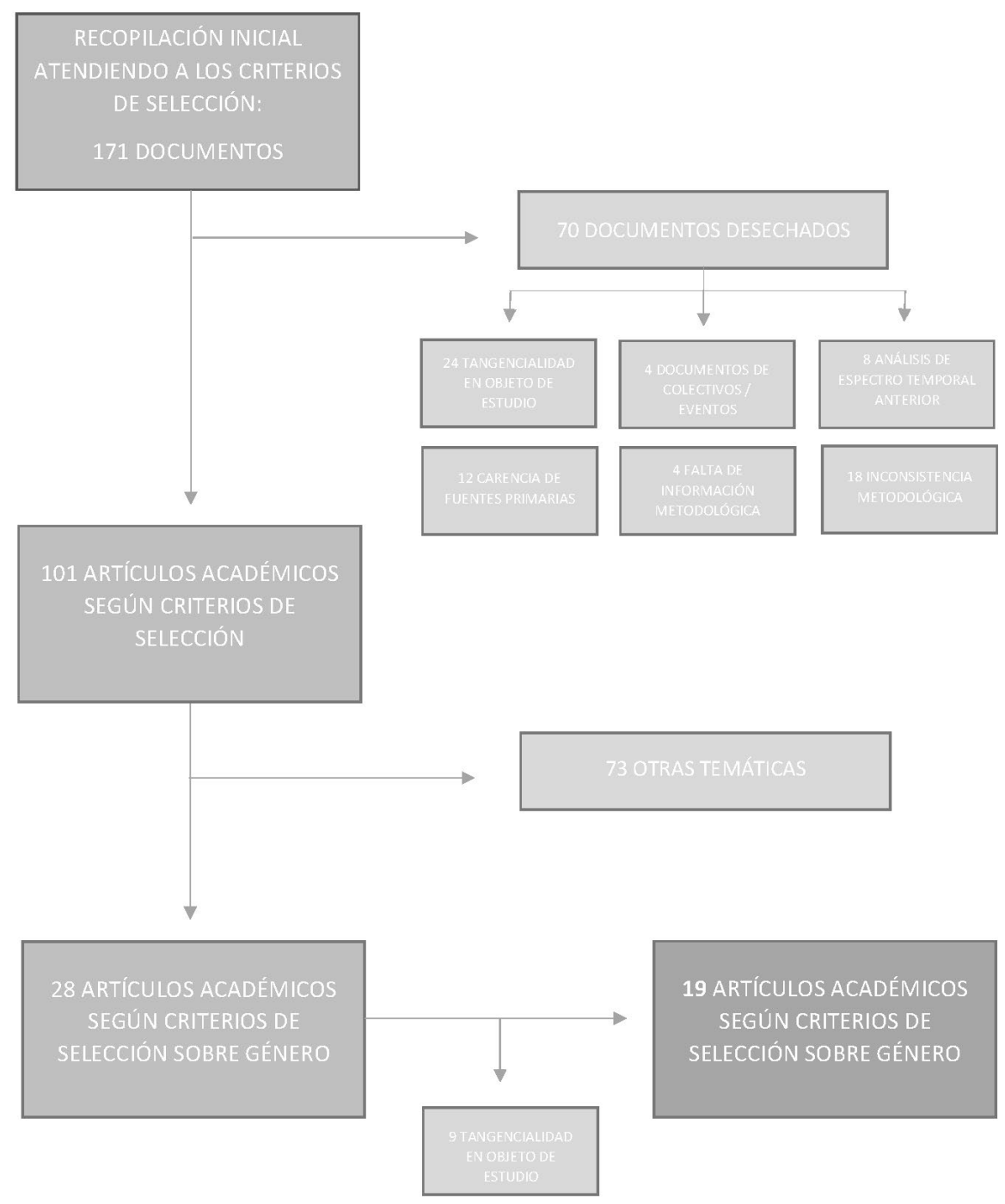

Feminismo/s 39, January 2022, 211-240 


\section{RESULTADOS}

\subsection{Perspectiva y análisis de género en los movimientos sociales en el contexto español durante el periodo 2010-2019}

Si analizamos las temáticas abordadas en los diferentes artículos de la primera muestra general de 101, vemos que el análisis del movimiento $15 \mathrm{M}$ se presenta como un claro punto de inflexión, al igual que lo fue en cuanto a la participación (general, y en particular femenina) en movimientos sociales y las nuevas dinámicas y canales utilizados para la protesta. A pesar de ello, siguen siendo escasas las investigaciones de carácter empírico, así como aquellas que analizan el proceso desde dentro o que tienen en cuenta su recorrido histórico (Alberich, 2019). Este interés por el 15M alcanza su máxima expresión en 2015, con el incremento del número de investigaciones centradas en la participación online, la vivienda o los feminismos. Posteriormente, en las 23 publicaciones de 2019, cuando aún aparecen referencias al 15M, destacan claramente los artículos sobre vivienda (6 de 23) y feminismo (8 de 23).

Así mismo, en los últimos años ha aumentado la diversidad temática dentro del estudio de los movimientos sociales, que se centran, además de en los feminismos y el activismo virtual, cuya transversalidad y diversidad de aplicaciones los conjuga muchas veces con otras temáticas, en el propio 15M, la vivienda, el movimiento vecinal/comunitario, la conflictividad laboral y el asociacionismo, así como en cuestiones más específicas como la participación política, la inmigración o el movimiento LGTBI.

Por último, y entrando ya en la cuestión de género, un dato especialmente relevante para la presente investigación es el hecho de que el número de autoras firmantes de los 101 artículos es superior al de autores (107 frente a 84), lo que viene a mostrar no solamente la creciente incorporación de las mujeres al ámbito académico e investigador dentro de las Ciencias Sociales (Betancor et al., 2019), sino también su mayor implicación con la movilización, la protesta y el activismo como formas de acción política en busca del cambio social. Esta situación no impide, sin embargo, que la perspectiva de género continúe siendo utilizada de forma muy reducida en este campo de estudio, pudiendo afirmarse, incluso, que es en realidad inexistente salvo en aquellos estudios en los que la propia participación femenina en los movimientos sociales forma parte del objeto central de la investigación. 
Perspectiva y análisis de género en las investigaciones sobre movimientos sociales y feminismos en el contexto español: una revisión sistemática

En concreto, el análisis final aquí presentado se delimita a los 19 artículos que abordan la cuestión del género de manera central en sus objetos de estudio (otros 9 lo hacían pero de forma tangencial, motivo por el que no han sido incluidos en esta fase específica).

Tabla 1. Muestra final analizada

\begin{tabular}{|c|c|c|c|c|}
\hline N. ${ }^{\circ}$ & Referencia & Año & Revista & Temática \\
\hline 1 & $\begin{array}{l}\text { Barreiro González, M. }{ }^{a} \text { Soliña y } \\
\text { Fernàndez i Aragonès, Aina }\end{array}$ & 2019 & $\begin{array}{l}\text { Sociología del } \\
\text { Trabajo }\end{array}$ & $\begin{array}{l}\text { Conflictividad } \\
\text { laboral }\end{array}$ \\
\hline 2 & $\begin{array}{l}\text { Bermúdez Figueroa, Eva y Roca } \\
\text { Martínez, Beltrán }\end{array}$ & 2019 & $\begin{array}{l}\text { Sociología del } \\
\text { Trabajo }\end{array}$ & Sindicalismo \\
\hline 3 & S. Cota, Ariana & 2019 & Papeles del CEIC & $\begin{array}{l}\text { Lucha } \\
\text { antirrepresiva }\end{array}$ \\
\hline 4 & $\begin{array}{l}\text { Núñez Puente, Sonia y } \\
\text { Fernández Romero, Diana }\end{array}$ & 2019 & $\begin{array}{l}\text { Investigaciones } \\
\text { feministas }\end{array}$ & Abuso sexual \\
\hline 5 & Romeo Echeverría, Aitor & 2019 & Encrucijadas & $\begin{array}{l}\text { Gestación } \\
\text { subrogada }\end{array}$ \\
\hline 6 & $\begin{array}{l}\text { Sádaba, Igor y Barranquero, } \\
\text { Alejandro }\end{array}$ & 2019 & Athenea Digital & Ciberfeminismo \\
\hline 7 & $\begin{array}{l}\text { Ballesteros, Ana; Franco, Sonia; } \\
\text { Donayre, Miguel y Serrano, Pilar }\end{array}$ & 2018 & Prisma social & $\begin{array}{l}\text { Participación } \\
\text { social }\end{array}$ \\
\hline 8 & Galdón Corbella, Carmen & 2018 & Encrucijadas & $\begin{array}{l}15 \mathrm{M} / \\
\text { Intergeneraciones }\end{array}$ \\
\hline 9 & Martínez, María & 2018 & Athenea Digital & Identidad colectiva \\
\hline 10 & Alonso, Alba y Paleo, Natalia & 2017 & RES & $\begin{array}{l}\text { Políticas de } \\
\text { igualdad }\end{array}$ \\
\hline 11 & Gandarias Goikoetxea, Itziar & 2017 & $\begin{array}{l}\text { Investigaciones } \\
\text { feministas }\end{array}$ & Interseccionalidad \\
\hline 12 & Martínez-Palacios, Jone & 2017 & $\begin{array}{l}\text { Revista Española } \\
\text { de Ciencia Política }\end{array}$ & $\begin{array}{l}\text { Participación } \\
\text { social }\end{array}$ \\
\hline 13 & $\begin{array}{l}\text { Royo, Raquel; Silvestre, María; } \\
\text { González, Lía; Linares, Estibaliz } \\
\text { y Suárez, Maialen }\end{array}$ & 2017 & $\begin{array}{l}\text { Investigaciones } \\
\text { feministas }\end{array}$ & Asociacionismo \\
\hline 14 & $\begin{array}{l}\text { Sanz Hernández, Alexia y López } \\
\text { Rodríguez, M. }{ }^{a} \text { Esther }\end{array}$ & 2017 & Aposta & $\begin{array}{l}\text { Conflictividad } \\
\text { laboral }\end{array}$ \\
\hline 15 & $\begin{array}{l}\text { Rivero Santamarina, Diana y } \\
\text { Larrondo Ureta, Ainara }\end{array}$ & 2016 & Teknokultura & Ciberfeminismo \\
\hline
\end{tabular}




\begin{tabular}{|c|l|c|l|l|}
\hline 16 & Trujillo, Gracia & 2016 & Encrucijadas & $\begin{array}{l}15 \mathrm{M} / \text { Activismos } \\
\text { queer }\end{array}$ \\
\hline 17 & Azpiazu Carballo, Jokin & 2015 & Papeles del CEIC & $\begin{array}{l}\text { Nuevas } \\
\text { masculinidades }\end{array}$ \\
\hline 18 & Ruiz García, Sonia & 2015 & $\begin{array}{l}\text { Revista Española } \\
\text { de Ciencia Política }\end{array}$ & $\begin{array}{l}\text { Relaciones } \\
\text { laborales }\end{array}$ \\
\hline 19 & Rodríguez García, María Jesús & 2013 & REIS & Asociacionismo \\
\hline
\end{tabular}

Nos encontramos, en este caso, con un aumento de las publicaciones en el año 2017, siendo en 2019 cuando se da el mayor número de artículos (6). De igual forma, destacan muy mayoritariamente las mujeres como autoras (81\% de la autoría), siendo, en proporción, una diferencia mucho mayor a la observada sobre el volumen total de publicaciones analizadas, donde representaban un 56\%. Las líneas temáticas abordadas en estos 19 artículos son diversas, destacando el estudio de las relaciones entre el género y el ámbito laboral con 4 publicaciones, y el asociacionismo y la participación social también con 4 publicaciones.

Se hace necesario aclarar en este punto la diferenciación realizada en el proceso de análisis entre aquellos artículos que aplican la perspectiva de género al estudio de una temática general y los que analizan el movimiento feminista en particular desde la perspectiva de género (véase tabla de resultados). No obstante, esta distinción se plantea de una forma más teórica que práctica, ya que su aplicación, a la hora de etiquetar en una u otra categoría las investigaciones analizadas, no es sencilla. Esta escasa claridad se evidencia, por ejemplo, en algunos casos en los que, partiendo desde el estudio de una temática general, se termina analizando el propio movimiento feminista generado en su seno. De igual forma, dada la interseccionalidad tanto teórica como social que esta corriente ha alcanzado en la actualidad (cuestión que algunos de los artículos abordan), su delimitación con otras áreas de estudio se hace cada vez más difícil.

\subsection{Acercamiento metodológico}

En cuanto a la cuestión metodológica, podemos ver cómo ninguna de las investigaciones utiliza metodología mixta, siendo prácticamente todas ellas abordadas desde un análisis cualitativo (tan sólo 2 publicaciones son estudios 
cuantitativos). La complementariedad de varias técnicas también es reducida, utilizándose en la mayor parte tan sólo una o dos, en especial la entrevista en profundidad, seguida de las fuentes documentales, la observación directa o participante y los grupos de discusión.

A la hora de publicar los resultados de un estudio primario, es recomendable proporcionar una explicación autocrítica de cómo se desarrolló metodológicamente la investigación (Seale, 1999). En este sentido, la transparencia metodológica resulta un instrumento muy adecuado para evaluar de forma sistemática la calidad y relevancia de los estudios, especialmente de aquellos de carácter cualitativo. Para analizarla en la presente revisión, hemos incluido una serie de criterios propios a partir de los establecidos en el método ENTREQ, utilizado como guía para aumentar la transparencia en la presentación de informes de investigación cualitativa en materia de salud (Tong et al., 2012). En la Tabla 2 pueden observarse los resultados del análisis conjunto de las investigaciones seleccionadas en nuestra muestra final. Se destaca de forma positiva la mayoritaria especificación en metodología y muestra, y como aspectos negativos el escaso reconocimiento de las limitaciones de los estudios y la reducida aplicación práctica de sus resultados. No obstante, cabe recordar que los artículos aquí analizados cuentan con un alto nivel de calidad al ser fruto de una selección en la que entre sus criterios se encontraban muchas de las cuestiones planteadas.

Tabla 2. Análisis de transparencia metodológica

\begin{tabular}{|l|c|c|c|}
\hline & Sí & $\begin{array}{c}\text { No de forma } \\
\text { clara }\end{array}$ & No \\
\hline ¿Se formula/n pregunta/s de investigación? & 5 & 7 & 7 \\
\hline ¿Se especifica/n objetivo/s? & 5 & 8 & 6 \\
\hline ¿Se explica exhaustivamente la metodología? & 12 & 5 & 2 \\
\hline ¿Se especifica la temporalidad? & 8 & 6 & 5 \\
\hline ¿Se concreta el volumen de la muestra? & 12 & 5 & 2 \\
\hline ¿Se explica la forma de análisis de los datos? & 7 & 7 & 5 \\
\hline ¿Se mencionan las limitaciones del estudio? & 5 & 4 & 10 \\
\hline $\begin{array}{l}\text { ¿Las conclusiones aportan conocimiento a su } \\
\text { campo de estudio? }\end{array}$ & 10 & 9 & 0 \\
\hline $\begin{array}{l}\text { ¿Se incluyen propuestas o aplicaciones } \\
\text { prácticas? }\end{array}$ & 3 & 7 & 9 \\
\hline
\end{tabular}

Feminismo/s 39, January 2022, 211-240 
Por otra parte, con respecto a la clasificación de los artículos por su campo de estudio, la investigación de los movimientos sociales se ha venido orientando históricamente en tres direcciones: a nivel externo (cuándo se activan), a nivel interno (qué hacen, cómo y por qué) y en función de su impacto (para qué) (Ibarra et al., 2002). Atendiendo a estos tres campos de análisis, se observa cómo la mayor parte de las investigaciones que forman parte de la muestra de la presente revisión sistemática (12) se centran sobre todo en el nivel interno de los movimientos que son su objeto de estudio. De esta forma, su interés se dirige mayoritariamente a la perspectiva y vivencia de las propias personas que los conforman, a nivel tanto individual como grupal, pero siempre desde su seno. A pesar de ello, también nos encontramos con algunas publicaciones que tratan de vislumbrar el impacto de dichos movimientos, no sólo en sus protagonistas, sino también en la transformación de la sociedad hacia posiciones más igualitarias entre hombres y mujeres, mostrando nuevas formas de acción y relación que van suponiendo cambios en los roles establecidos para cada género. Tan sólo dos de las investigaciones analizadas estudian los procesos de surgimiento y desarrollo a nivel externo.

\subsection{Revisión temática y de contenidos}

En cuanto al propio análisis de los resultados obtenidos en las diferentes investigaciones seleccionadas, se han detectado varias cuestiones en común y que son abordadas de forma reiterada, incluso desde puntos temáticos de partida diferentes (véase tabla de resultados).

Por una parte, la percepción de posiciones de desigualdad e invisibilización de las mujeres aún vigentes en los diferentes ámbitos de estudio de la mayor parte de las investigaciones es el punto de partida de prácticamente todos los artículos (de forma más o menos explícita). De esta manera, una de las principales y más básicas conclusiones de los artículos es que sigue existiendo aún mucha desigualdad en cuestión de género en los propios movimientos sociales (Barreiro y Fernàndez, 2019; Bermúdez y Roca, 2019; Sanz y López, 2017). A pesar de ello, también es reconocido de forma habitual el empuje que el 15M tuvo para el movimiento feminista (Galdón, 2018), aumentando progresivamente desde entonces, como se ha visto con el conjunto de publicaciones analizadas. 
Es precisamente a partir de la conquista y re-conquista de espacios desde donde se perciben procesos de transformación, tanto a nivel individual o social como político, que rompen los márgenes de lo establecido, generan espacios de participación más igualitarios y desarrollan nuevas formas de acción para las mujeres, al tiempo que se ponen en valor las clásicamente feminizadas (Azpiazu, 2015; Ballesteros et al., 2018; Barreiro y Fernàndez, 2019; Cota, 2019; Martínez-Palacios, 2017; Rodríguez, 2013; Ruiz, 2015; Royo et al., 2017; Sádaba y Barranquero, 2019; Sanz y López, 2017).

Por otro lado, y de forma más concreta, entre las conclusiones de los artículos analizados surgen algunos de los valores y principios básicos de las tendencias que de alguna forma están marcando la particularidad del movimiento feminista de la última década. Así, muchas de las autoras apuestan, a partir de los resultados de sus estudios, por la horizontalidad y la sororidad (Ballesteros, 2018; Ruiz, 2015) como estrategias base del movimiento para su unidad hacia el exterior, en contraposición a las múltiples disidencias percibidas en su interior sobre algunas cuestiones clave como puede ser la maternidad subrogada, provocando rupturas entre las diferentes perspectivas al respecto (Alonso y Paleo, 2017; Ballesteros et al., 2018; Cota, 2019; Galdón, 2018; Martínez, 2018; Romeo, 2019; Ruiz, 2015; Trujillo, 2016).

Con ello, una de las ideas clave reflejadas en las investigaciones analizadas es la percepción de la interseccionalidad y transversalidad de género como aspectos imprescindibles para alcanzar el objetivo de construir una sociedad igualitaria, no sólo frente al género, sino frente a todas las demás discriminaciones que le son transversales (Ballesteros et al., 2018; Cota, 2019; Galdón, 2018; Gandarias, 2017; Martínez, 2018; Royo et al., 2017; Trujillo, 2016).

Así surge la cuestión de la/s identidad/es como uno de los ejes centrales de muchos de los análisis (Azpiazu, 2015; Barreiro y Fernàndez, 2019; Galdón, 2018; Martínez, 2018; Sádaba y Barranquero, 2019; Sanz y López, 2017), atendiendo a los procesos de conformación de la identidad tanto hacia dentro, a nivel individual, como hacia afuera, a nivel grupal. La relevancia que para todos los movimientos sociales tiene el compartir identidades colectivas que se conformen como claves del movimiento para su re-identificación grupal, o el propio refuerzo que la socialización en estos espacios puede darles, entran en juego como un elemento vital en los procesos de 
transformación ya mencionados (Gandarias, 2017; Martínez, 2018; Sanz y López, 2017).

En relación con esto nos encontramos ante un interesante debate sobre los dilemas ético-políticos del acercamiento al estudio de los movimientos sociales a través de un análisis reflexivo y situado mediante la articulación de la investigación científica y el activismo social, siendo cada vez más habitual este tipo de perfil mixto de activista/investigadora o investigadora/ activista (Cota, 2019; Trujillo, 2016). Pese a la controversia que puede generar la investigación militante o "participación implicada» (Cota y Sebastiani, 2015), numerosos/as autores/as y sus trabajos reflejan cómo la combinación del compromiso crítico con la investigación activista abre la puerta a nuevas posibilidades y horizontes (Arribas, 2014), produciendo un conocimiento que contribuye no solamente al mundo académico, sino también a la lucha por la justicia social. Esta relación híbrida entre académicos/as y activistas, que ha ido aumentando en los últimos años en España, se encuentra especialmente presente en el análisis de los feminismos, constituyendo lo que se conoce como «investigación feminista activista» (Biglia, 2007), algo que se refleja en nuestra muestra de estudio, donde en 4 de los artículos las propias investigadoras se identifican también como parte del movimiento que analizan. En todo caso, esta complementariedad de ambos perfiles, a pesar de no ser indicada de forma explícita, puede inferirse de forma implícita en muchas otras publicaciones por el tipo de movimiento que analizan y las técnicas utilizadas, como la observación participante. Cabría aquí reflexionar sobre hasta qué punto analizar el movimiento feminista desde una perspectiva de género no es pertenecer ya a él, en mayor o menor medida (al menos de manera teórica).

\subsection{La particularidad de los ciberfeminismos}

Otra de las cuestiones comunes en varias de las publicaciones analizadas, y que se anticipaba en el apartado teórico, es la atención a nuevas formas y narrativas de participación femenina dentro de los movimientos sociales, que se unen y mezclan con otras clásicas aún vigentes. Además de la ya consolidada participación online, se están desarrollando cada vez en mayor medida nuevas formas de protesta ligadas al concepto de «creatividad social» 
(Calle, 2000), apostando por las reivindicaciones a través de lo simbólico y no sólo de lo material. En estos casos las demandas son llenadas de contenido (Barreiro y Fernàndez, 2019) al promover y hacer uso de la cultura libre en la que la base es el código abierto, la ausencia de límites (Galdón, 2018).

De esta forma, las redes sociales y los espacios web se conforman como relevantes puntos de encuentro y relación en los que poner en común diversas experiencias y visiones. Esta esfera digital permite no sólo visibilizar, sino también incrementar el impacto de algunas de las luchas y demandas del movimiento, aportando un mayor alcance de sus mensajes al poder ser transmitidos por todo el mundo de forma inmediata. Es decir, la dimensión virtual ejerce de altavoz, pudiendo transferir las demandas de las calles a otros espacios y actores sociales a través de su difusión masiva. En suma, conforma una estrategia mediática para la promoción del movimiento feminista.

Sin embargo, las investigadoras no perciben una verdadera reflexión ni un debate profundo de trasfondo sobre las cuestiones estructurales del movimiento feminista y sus demandas de actualidad (Núñez Puente et al., 2016). Esto supone un arma de doble filo al dar lugar al surgimiento de múltiples perspectivas y posicionamientos de forma rápida y libre, no siempre construidos de forma activa. Este fenómeno ha sido llamado «movilización efímera», escondiendo bajo una ilusión de participación acciones de carácter puntual, de mero consumo pasivo, no reflexivo, y con un alcance real menor al considerado (Rodríguez-Suárez et al., 2021), y conforma uno de los principales riesgos de la acción social en red.

Es aquí donde se encuentran los denominados «contramovimientos»y la «posverdad», generando bulos y noticias falsas desde posturas contrarias, con el fin de desacreditar los mensajes y reivindicaciones que se tratan de evidenciar. No obstante, también se puede caer en estas acciones desde el propio movimiento, dando lugar a ciertos procesos de revictimización hacia las mujeres y generando posicionamientos pasivos de las mismas, algo que es percibido por algunos colectivos (Alonso y Paleo, 2017; Barreiro y Fernàndez, 2019; Núñez y Fernández, 2019; Rivero y Larrondo, 2016). A pesar de ello, en ocasiones estas esferas son el punto de partida de grandes movimientos sociales en red, que conforman «redes de indignación y esperanza», en palabras de Castells (2012), que conectan las calles con los flujos globales de indignación. En definitiva, a pesar de los retos del cariz diluido de la 
participación en red, desde la difusión de convocatorias hasta la socialización de contenidos gráficos para su refuerzo, las redes sociales han sido y son utilizadas como vehículo para la difusión de los mensajes y reivindicaciones feministas, aumentando su alcance y facilitando su expansión.

A partir de todo ello se llega, como conclusión del conjunto de las publicaciones, al reconocimiento de la pluralidad existente tanto dentro del movimiento feminista como en toda la participación femenina en la actualidad, visible al observar las diferentes temáticas que cada una de ellas aborda y pudiendo extrapolarse para representar a aquellos estudios que no han sido analizados o mencionados por su particularidad (como es el caso de la juventud, el colectivo estudiantil, las personas mayores, migrantes, pertenecientes al ámbito rural, etc.).

En definitiva, la pluralidad de los estudios analizados puede servir como reflejo de la diversidad que caracteriza y define a los actuales movimientos feministas, en el momento en que, paradójicamente, han logrado movilizarse tras las mismas pancartas (Galdón, 2019). No obstante, resulta necesario recordar que, si bien hoy en día suponen de forma indiscutible el mayor de los movimientos sociales en cuanto a activismo femenino, el feminista no es el único ámbito en que las mujeres se movilizan ni puede, por tanto, generalizarse a toda la participación social femenina.

\section{CONCLUSIONES}

La presente revisión sistemática ha permitido obtener una visión panorámica de los estudios empíricos centrados en los movimientos sociales desde una perspectiva de género y/o en los feminismos, desarrollados en la última década en el contexto español.

Conviene, sin embargo, reconocer las propias limitaciones del presente trabajo. Así, sería de gran interés incluir en una futura ampliación un mayor campo de análisis, tanto temporal, lo que permitiría obtener una visión más completa de la evolución de los movimientos sociales y su estudio, como geográfico, aplicando esta metodología a otros contextos concretos dentro del ámbito español o fuera de sus fronteras. De igual modo, trabajar con una muestra más amplia de investigaciones empíricas aportaría mayor solidez a la revisión y sus resultados. Para ello se podría analizar otras revistas no 
tenidas en cuenta en la presente revisión, así como desarrollar una nueva búsqueda sistemática en diferentes bases de datos de otro tipo de publicaciones (libros, capítulos de libro, tesis doctorales...).

En todo caso, la revisión sistemática realizada nos ha permitido extraer conclusiones relevantes acerca del objeto de estudio. En primer lugar, y de forma general, si ya es echado en falta un mayor número de investigaciones centradas en la movilización social en nuestro país, esta carencia es aún mayor en el caso de aquellas llevadas a cabo desde la perspectiva de género. Así, se ha constatado una gran limitación a la hora de incorporar esta mirada a la investigación en esta materia, encontrándose prácticamente de forma exclusiva cuando el propio objeto de estudio se centraba en el activismo femenino y/o feminista.

En este sentido, dentro de las investigaciones analizadas se observa una gran deficiencia en la aplicación de una perspectiva de género que tenga en cuenta las particularidades de las oportunidades y acceso de las mujeres a la participación social, así como los contenidos y formas de su acción. Es por esto por lo que consideramos imprescindible aplicar la perspectiva de género de manera transversal a la investigación sea cual sea la temática de estudio y, en este caso, se analice un movimiento social mayoritariamente femenino o no. La incorporación de esta mirada es precisa no solamente al profundizar en el propio movimiento feminista (movilización femenina por antonomasia), sino en todos los espacios y movimientos sociales en que la intervención femenina ha estado y todavía está invisibilizada, limitada y opacada por «lo masculino». Esto pasa por reconocer a los movimientos feministas como objeto de estudio a un nivel equiparable a la magnitud que están adquiriendo, tanto a nivel nacional como internacional, como agentes de cambio social. Por ello, creemos necesario incidir en la necesidad de que, tanto desde el ámbito institucional como desde los propios medios de difusión de conocimiento, se apoye y promueva el estudio de los movimientos sociales y, en especial, de aquellos llevados a cabo desde una perspectiva de género o centrados en los feminismos.

Por otro lado, se ha identificado que la muestra general, a diferencia de los estudios centrados en la perspectiva de género y feminista, cuenta con un escaso análisis de las dimensiones psicosociales de la participación, que apenas atiende a las particularidades de la participación femenina, 
incidiéndose, en la mayor parte de los casos, en una visión enfocada en las causas y consecuencias de los movimientos en general, como un todo, más que a su vivencia interna, tanto individual como colectiva. No obstante, es preciso poner en valor también aquellas investigaciones que sitúan en el centro de sus análisis los procesos de transformación que tienen lugar en el seno de estos espacios con base en identidades compartidas y de construcción colectiva. Éstas permiten mostrar la parte menos visible en el ámbito académico de los movimientos sociales, así como el papel que las propias investigadoras y sus relaciones con el espacio juegan en este sentido. Con ello cabe destacar que en general, y particularmente en las investigaciones de carácter feminista, se sigue utilizando de forma reiterativa el término «empoderamiento» para hacer referencia a procesos colectivos de visibilidad y disidencia con lo impuesto, cuando, en realidad, más que de un cambio en los propios agentes, se trata de una transformación de su contexto y su entorno, de los espacios y las condiciones de desarrollo en ellos.

Así mismo, atendiendo a los resultados de las investigaciones de género y feministas realizadas en los últimos años en el ámbito de la movilización social, se observan trabajos básicamente descriptivos, echándose en falta estudios de los que puedan derivarse propuestas interventivas o de aplicación práctica. Su realización permitiría ir más allá del mero conocimiento del terreno, posibilitando una mejor integración y un desarrollo más adecuado de la participación social y colectiva de las mujeres. Esta forma de investigación aplicada podría conseguirse aumentando el uso de metodología mixta, inexistente en la muestra analizada, así como la complementariedad de técnicas, lo que facilitaría un conocimiento más amplio y diverso del ámbito de estudio, dando pistas sobre las líneas de desarrollo posibles. Del mismo modo, aumentar el tamaño de las muestras y el alcance de las investigaciones favorecería también esta tarea.

Para finalizar, cabe destacar, como puntos fuertes y potencialidades, la diversidad y pluralidad que caracterizan a las investigaciones existentes sobre movimientos sociales con perspectiva de género y/o centradas en los feminismos. Así, se analizan diferentes temáticas, tanto tradicionales como novedosas, al tiempo que se indaga en las nuevas formas y narrativas de la participación femenina colectiva, lo que ayuda a conformar un mejor conocimiento general de este campo de estudio, muy reducido hasta el momento. 
Es el caso de la atención a los crecientes ciberfeminismos y, con ello, a las particularidades y retos que se presentan ante la acción femenina en los espacios virtuales. Del mismo modo, se rescata en positivo el creciente interés de la comunidad científica por este campo de estudio en los últimos años, en especial por parte de las investigadoras sociales, situándose a la par del desarrollo de los acontecimientos causales y consecuenciales de la acción social en nuestro país. Esto nos permite pronosticar una continuación de esta tendencia en el futuro, ampliándose no sólo el número de investigaciones al respecto, sino también la diversidad de áreas como objeto de estudio y el uso de diferentes técnicas metodológicas para la consecución de sus objetivos.

\section{REFERENCIAS}

Adell, R. (2000). Movimientos sociales en los años noventa: volumen, actores y temas de movilización. En E. Grau y P. Ibarra (Coords.), Una mirada sobre la red. Anuario de movimientos sociales (pp. 27-52). Fundación Betiko.

Aguilar, S. (2001). Movimientos sociales y cambio social. ¿Una lógica o varias lógicas de acción colectiva? Revista Internacional de Sociología, 30, 29-62. https://doi.org/10.3989/ris.2001.i30.767

Ahmed, S. (2018). Vivir una vida feminista. Bellaterra.

Alberich, T. (2019). ¿Y después del 15M, qué? Ciclos de movilización social y aprendizajes conceptuales. En R. Díaz García y G. Betancor Nuez (Eds.), Movimientos sociales, acción colectiva y cambio social en perspectiva. Continuidades y cambios en el estudio de los movimientos sociales (pp. 17-30). Fundación Betiko.

Alonso, A. y Paleo, N. (2017). Políticas de salud sexual y reproductiva en España: Contra-movimientos y marcos interpretativos en conflicto. Revista Española de Sociología, 26(3 Supl.), 59-76. https://doi.org/10.22325/fes/res.2017.35

Arribas, A. (2014). Lógicas emergentes de acción colectiva y prácticas colaborativas de investigación. Apuntes para una Antropología junto y con los movimientos sociales. Gazeta de Antropología, 30(1), artículo 07. http://hdl. handle.net/10481/30775

Astudillo-Mendoza, P. A., Figueroa-Quiroz, V. A. y Cifuentes-Zunino, F. (2020). Navegando entre mujeres: la etnografía digital y sus aportes a las investigaciones feministas. Investigaciones feministas, 11(2), 239-249. https://doi. org/10.5209/infe. 65878 
Azpiazu Carballo, J. (2015). Men and feminism: from the privilege of comfort to the uneasiness of political engagement in feminist issues. Papeles del CEIC, 2015/2(127). https://doi.org/10.1387/pceic.14163

Ballesteros Pena, A., Franco Alonso, S., Donayre Pinedo, M. y Serrano Garijo, P. (2018). La evaluación del Proyecto Lideresas del Ayuntamiento de Madrid: Una experiencia de evaluación desde la teoría del programa sensible al género y los Derechos Humanos. Prisma Social, 21, 357-390. https://revistaprismasocial.es/article/view/2437

Barreiro González, M. S. y Fernàndez i Aragonès, A. (2019). Infrarrepresentación y distorsión de la identidad en los medios durante los conflictos laborales: El caso de la huelga de las trabajadoras de Bershka. Sociología del Trabajo, 95, 105-123. https://doi.org/10.5209/stra.66445

Bermúdez Figueroa, E. y Roca Martínez, B. (2019). Participación de mujeres en el movimiento sindical. Análisis desde la perspectiva de los recursos de poder. Sociología del Trabajo, 95, 53-72. https://doi.org/10.5209/stra.66435

Betancor, G., Díez, R., Tejerina, B., Funes, M. J. y Adell, R. (2019). El campo de estudio de los movimientos sociales en España desde una perspectiva longitudinal. En R. Díaz García y G. Betancor Nuez (Eds.), Movimientos sociales, acción colectiva y cambio social en perspectiva. Continuidades y cambios en el estudio de los movimientos sociales (pp. 193-219). Fundación Betiko.

Biglia, B. (2007). Desde la investigación-acción hacia la investigación activista feminista. En J. Romay Martínez (Coord.), Perspectivas y retrospectivas de la psicología social en los albores del siglo XXI (pp. 415-422). Biblioteca Nueva. Calle, A. (2000). Ciudadanía y solidaridad. IEPALA.

Castells, M. (2012). Redes de indignación y esperanza. Alianza Editorial.

Cook, D. J., Sackett, D. L. y Spitzer, W. O. (1995). Methodologic guidelines for systematic reviews of randomized control trials in health care from the Potsdam Consultation on Meta-Analysis. Journal of Clinical Epidemiology, 48, 167-71. https://doi.org/10.1016/0895-4356(94)00172-M

Cota, A. S. (2019). Procesos de agenciamiento junto a Stop Represión Granada y un ejercicio de autoetnografía vulnerable. Papeles del CEIC, 2019/1(207), 1-19. https://doi.org/10.1387/pceic.19530

Cota, A. S. y Sebastiani, L. (2015). «Que no, que no, que no nos representan», o repensando la relación entre investigación y activismo a partir de nuestras experiencias vividas. Ankulegi, 19, 43-58. 
Delgado-López-Cózar, E. (2020). El ranking de revistas científicas españolas con Sello de calidad Fecyt: un constructo bibliométrico artificioso y obsoleto. Anuario ThinkEPI, 14, el4e01. https://doi.org/10.3145/thinkepi.2020.e14e01 Di Liscia, M. H. B. (2007). Memorias de mujeres. Un trabajo de empoderamiento. Política y Cultura, 28, 43-69.

Díez, R. (2019). Sociedad civil y movimientos sociales. Entre el cambio y la organización social. Revista Española de Sociología, 28(1), 161-169. https:// doi.org/10.22325/fes/res.2018.55

Dunezat, X. (2017). Dominación masculina y feminismo en los movimientos sociales. Política y Sociedad, 54(2), 399-419. https://doi.org/10.5209/ POSO. 52740

Folguera, P. (1998). El feminismo en España: dos siglos de historia. Editorial Pablo Iglesias.

Galdón Corbella, C. (2018). Cosmovisiones feministas en clave generacional. Del movimiento 15M a la Huelga Feminista del 8M. Encrucijadas. Revista Crítica de Ciencias Sociales, 16(v1602).

Galdón Corbella, C. (2019). Del movimiento 15M a la huelga feminista del 8M. Un recorrido y algunas claves para entender el presente del movimiento feminista. En R. Díaz García y G. Betancor Nuez (Eds.), Movimientos sociales, acción colectiva y cambio social en perspectiva. Continuidades y cambios en el estudio de los movimientos sociales (pp. 87-100). Fundación Betiko.

Gandarias Goikoetxea, I. (2017). ¿Un neologismo a la moda?: Repensar la interseccionalidad como herramienta para la articulación política feminista. Investigaciones Feministas, 8(1), 73-93. https://doi.org/10.5209/INFE.54498 Goldsmith, M. (2002). Feminismo e investigación social. Nadando en aguas revueltas. En E. Bartra (Comp.), Debates en torno a una metodología feminista (pp. 35-62). Universidad Autónoma Metropolitana.

Grau Biosca, E. (1993). De la emancipación a la liberación y la valoración de la diferencia. El movimiento de mujeres en el Estado español. 1965-1990. En G. Duby y M. Perrot (Dirs.), Historia de las mujeres en Occidente. El siglo XX (pp. 673-683). Taurus.

Higgins, J. P. y Green, S. (Eds.) (2011). Cochrane handbook for systematic reviews of interventions (Vol. 4). John Wiley \& Sons.

Ibarra Güell, P., Martí i Puig, S. y Gomà Carmona, R. (Coords.) (2002). Creadores de democracia radical. Movimientos sociales y redes de políticas públicas. ICARIA. 
Íñiguez, L. (2003). Movimientos sociales: conflicto, acción colectiva y cambio social. En F. Vázquez (Coord.), Psicología de la acción colectiva (pp. 75-134). EDIUOC.

Javaloy, F. (2003). Comportamiento colectivo y movimientos sociales: un reto para la Psicología Social. Revista de Psicología Social, 18(2), 163-206. https:// doi.org/10.1174/021347403321645267

Keller, E. F. (1991). Reflexiones sobre género y ciencia. Alfons el Magnànim.

Laraña, E. (1999). La construcción de los movimientos sociales. Alianza.

Martínez, M. (2018). Reiteraciones relacionales y activaciones emocionales: hacia una radicalización de la procesualidad de las identidades colectivas. Athenea Digital, 18(1), 293-317. https://doi.org/10.5565/rev/athenea.1860

Martínez-Palacios, J. (2017). Democratizing participation through feminism. The role of feminist subaltern counterpublics in the expansion of the Basque public sphere. Revista Española de Ciencia Política, 43, 37-59. https://doi. org/10.21308/recp.43.02

Ministerio de Ciencia e Innovación (2011). El género en la investigación. Comisión Europea, Gobierno de España. https://doi.org/10.2777/23655

Moher, D., Liberati, A., Tetzlaff, J., Altman, D. G. y The PRISMA Group (2009). Preferred reporting items for systematic reviews and meta-analyses: The PRISMA Statement. PLoS Medicine, 6(7:e1000097). https://doi.org/10.1371/ journal.pmed.1000097

Nicolás, G. (2009). Debates en epistemología feminista: del empiricismo y el standpoint a las críticas postmodernas sobre el sujeto y el punto de vista. En G. Nicolás, E. Bodelón, R. Bergalli e I. Rivera Beiras (Coords.), Género y dominación: críticas feministas del derecho y del poder (pp. 25-62). Anthropos. Núñez Puente, S. y Fernández Romero, D. (2019). Posverdad y victimización en Twitter ante el caso de La Manada: propuesta de un marco analítico a partir del testimonio ético. Investigaciones Feministas, 10(2), 385-398. https://doi. org/10.5209/infe.66501

Núñez Puente, S., Fernández Romero, D. y Peña Jiménez, P. (2016). Ciberactivismo contra la violencia de género: fetichismo tecnológico e interactividad. Feminismo/s, 27, 177-195. https://doi.org/10.14198/fem.2016.27.10

Pajares, L. (2020). Fundamentación feminista de la investigación participativa: Conocimiento, género y participación, o del diálogo necesario para la transformación. Investigaciones feministas, 11(2), 297-306. https://doi.org/10.5209/ infe. 65844 
Pantera Rosa (2004). Moverse en la incertidumbre. Dudas y contradicciones de la investigación activista. En M. Malo (Ed.), Nociones comunes. Experiencias y ensayos entre investigación y militancia (pp. 191-205). Traficantes de sueños. Pérez Ledesma, M. (1994). Cuando lleguen los días de la cólera. (Movimientos Sociales, Teoría e Historia). Zona Abierta, 69, 51-120.

Pertegal-Vega, M. A., Oliva-Delgado, A. y Rodríguez-Meirinhos, A. (2019). Revisión sistemática del panorama de la investigación sobre redes sociales: Taxonomía sobre experiencias de uso. [Systematic review of the current state of research on Online Social Networks: Taxonomy on experience of use]. Comunicar, 27, 81-91. https://doi.org/10.3916/C60-2019-08

Pleyers, G. y Álvarez-Benavides, A. (2019). La producción de la sociedad a través de los movimientos sociales. Revista Española de Sociología, 28(1), 141-149. https://doi.org/10.22325/fes/res.2018.53

Pont Vidal, J. (1998). La investigación de los movimientos sociales desde la sociología y la ciencia política. Una propuesta de aproximación teórica. Papers: Revista de Sociología, 56, 257-72. https://doi.org/10.5565/rev/papers.1955

Ramos, M. D. (2000). Identidad de género, feminismo y movimientos sociales en España. Historia contemporánea, 21, 523-552.

Requena, M. (2014). La evaluación de la investigación a debate. Revista Española de Sociología, 21, 129-136. https://recyt.fecyt.es/index.php/res/article/ view/65363

Rivero Santamarina, D. y Larrondo Ureta, A. (2016). La actividad de las publicaciones feministas en red: retos para la transmisión de la cultura feminista en España. Teknokultura, 13(1), 117-140. https://doi.org/10.5209/rev_TK.2016. v13.n1.52180

Rodríguez García, M. J. (2013). El pluralismo asociativo femenino en municipios españoles. Propuesta de tipología. Revista Española de Investigaciones Sociológicas, 142, 123-140. https://doi.org/10.5477/cis/reis.142.123

Rodríguez-Suárez, J., Morán-Neches, L. y Herrero-Olaizola, J. (2021). Online research, new languages and symbolism of digital activism: A systematic review. [Investigación en red, nuevos lenguajes y simbologías del activismo digital: Una revisión sistemática]. Comunicar, 68, 47-58. https://doi. org/10.3916/C68-2021-04

Romeo Echeverría, A. (2019). Gestación subrogada y movimiento feminista. Una aproximación cuantitativa. Encrucijadas. Revista Crítica de Ciencias Sociales, 18(a1806). 
Royo Prieto, R., Silvestre Cabrera, M., González Estepa, L., Linares Bahillo, E. y Suárez Errekalde, M. (2017). Mujeres migrantes tejiendo democracia y sororidad desde el asociacionismo. Una aproximación cualitativa e interseccional. Investigaciones Feministas, 8(1), 223-243. https://doi.org/10.5209/ INFE.54496

Ruiz García, S. (2015). Power and representation: feminist movement challenges on work. Revista Española de Ciencia Política, 39, 195-220.

Sádaba, I. y Barranquero, A. (2019). Las redes sociales del ciberfeminismo en España: identidad y repertorios de acción. Athenea Digital, 19(1), e2058. https://doi.org/10.5565/rev/athenea.2058

Sagastizabal, M. y Luxán, M. (2016). Género y uso del tiempo. En M. Legarreta (Ed.), Dos décadas de cambio social en la C.A. de Euskadi a través del uso del tiempo. Encuesta de Presupuestos de Tiempo 1993-2013 (pp. 385-423). Instituto Vasco de Estadística-Eustat.

Sánchez-Meca, J. (2010). Cómo realizar una revisión sistemática y un meta-análisis. Aula Abierta, 38(2), 53-64.

Sanz-Casado, E., Melero, R., Aleixandre-Benavent, R., Codina, L., CosladoBernabé, M. A., De-Filippo, D., Giménez-Toledo, E., Jiménez, E. y RicoCastro, P. (2020). Ranking de visibilidad e impacto de revistas científicas españolas de Humanidades y Ciencias Sociales con sello de calidad FECYT. Fundación Española para la Ciencia y la Tecnología.

Sanz Hernández, A. y López Rodríguez, M. E. (2017). Mujeres del carbón. Protestas y emociones en la reestructuración minera española. Aposta. Revista de Ciencias Sociales, 74, 84-110. http://apostadigital.com/revistav3/ hemeroteca/alexsanz.pdf

Seale, C. (1999). Quality in qualitative research. Qualitative Inquiry, 5(4), 465478. https://doi.org/10.1177/107780049900500402

Tejerina, B. (1998). Los movimientos sociales y la acción colectiva. En P. Ibarra y B. Tejerina (Eds.), Los movimientos sociales. Transformaciones politicas y cambio cultural (pp. 111-139). Trotta.

Tilly, Ch. (2005). La democratización mediante la lucha. Sociológica, 19(57), 35-59. https://doi.org/10.3989/asclepio.2005.v57.i2.58

Tong, A., Flemming, K., McInnes, E., Oliver, S. y Craig, J. (2012). Enhancing transparency in reporting the synthesis of qualitative research: ENTREQ. BMC Medical Research Methodology, 12(181), 1-8. https://doi. org/10.1186/1471-2288-12-181 
Trujillo, G. (2016). La protesta dentro de la protesta. Activismos queer/cuir y feministas en el 15M. Encrucijadas. Revista Crítica de Ciencias Sociales, 12(a1202).

Urrútia, G. y Bonfill, X. (2010). Declaración PRISMA: Una propuesta para mejorar la publicación de revisiones sistemáticas y metaanálisis. Medicina Clínica, 135(11), 507-511. https://doi.org/10.1016/j.medcli.2010.01.015 